\title{
Síndrome de burnout em profissionais de Enfermagem que atuam no setor de urgência e emergência
}

Burnout syndrome in Nursing professionals working in the urgency and emergency sector

El síndrome de burnout en los profesionales de Enfermería que trabajan en el sector de urgencias y emergencias

Fabricia Seixas dos Reis ORCID: https://orcid.org/0000-0002-8352-9107 Centro Universitário FAMETRO, Brasil E-mail: fabricia.reiis@gmail.com

Irlane Ferreira França

ORCID: https://orcid.org/0000-0003-0878-6776 Centro Universitário FAMETRO, Brasil

E-mail: irlanef84@gmail.com

Italo Everton Bezerra Barbosa

ORCID: https://orcid.org/0000-0002-0225-7569

Centro Universitário FAMETRO, Brasil

E-mail: italoeverton1998@gmail.com

Alicia Ribeiro Fonseca

ORCID: https://orcid.org/0000-0002-6904-3346 Centro Universitário FAMETRO, Brasil E-mail: alicia.ribeiro2013@gmail.com

Breno de Souza Mota

ORCID: https://orcid.org/0000-0003-1340-2204

Universidade de São Paulo, Brasil

E-mail: brenosouzamota@usp.br

Felipe Chrystian de Figueiredo Lira

ORCID: https://orcid.org/0000-0003-1820-5605

Centro Universitário FAMETRO, Brasil

E-mail: felipechrystianf.lira01@gmail.com

Fabrício de Souza Melo

ORCID: https://orcid.org/0000-0002-3856-7964

Fundação de Vigilância em Saúde do Estado do Amazonas, Brasil

E-mail: enf.fabricio.melo@gmail.com

Izabel Cruz da Rocha

ORCID: https://orcid.org/0000-0001-6379-685X Centro Universitário FAMETRO, Brasil

E-mail: belzinha.marie@gmail.com

Josilene Farias Moraes

ORCID: https://orcid.org/0000-0002-9808-1894 Centro Universitário FAMETRO, Brasil

E-mail: josilene.f.m@gmail.com

Letícia de Nazaré Costa Flexa

ORCID: https://orcid.org/0000-0002-3646-1322 Centro Universitário FAMETRO, Brasil

E-mail: leticiaflexa123456@gmail.com

Luísa Magalhães Rubem

ORCID: https://orcid.org/0000-0002-4070-5772 Centro Universitário FAMETRO, Brasil

E-mail: luisa.123.mr19@gmail.com

Misaele Silva Maciel

ORCID: https://orcid.org/0000-0002-8259-6578 Centro Universitário FAMETRO, Brasil

E-mail: misaelesilva55@gmail.com

Nataly Danielle Araújo Queiroz

ORCID: https://orcid.org/0000-0003-4853-9227

Líder Instituto Educacional, Brasil

E-mail: natty24queiroz@gmail.com

Pedro Jorge da Silva Pires

ORCID: https://orcid.org/0000-0001-5331-6146 Centro Universitário FAMETRO, Brasil E-mail: pjsilva009@gmail.com 


\author{
Valdirene Oliveira Avinte \\ ORCID: https://orcid.org/0000-0003-0198-7006 \\ Centro Universitário FAMETRO, Brasil \\ E-mail: valdireneavinte@outlook.com
}

\begin{abstract}
Resumo
Este estudo teve como objetivo realizar uma busca ativa na literatura sobre a SB nos profissionais de enfermagem que atuam na área de urgência e emergência, abordando em conjunto os principais preditores para o desenvolvimento da síndrome. Trata-se de estudo de origem descritivo, com a abordagem metodologia qualitativa, utilizando a Revisão Integrativa da Literatura (RIL) como técnica, em conjunto com estratégia de pico para formulação da pergunta norteadora. A pesquisa foi realizada entre os meses de abril e junho de 2021 nas bases indexadas dentro da Biblioteca Virtual de Saúde (BVS), sendo: (MEDLINE), (LILACS) e (BDENF), através da junção de três descritores em saúde (DeCS) cruzados com o operador booleano "AND": Esgotamento psicológico AND Enfermagem AND Trabalho, sendo encontrados 1.007 artigos no total. Após a aplicação dos critérios de publicações dentro dos idiomas português e inglês, dentro da linha temporal estabelecida nos últimos cinco anos (2016 a 2021), que tivessem correlação com a pergunta problema, foram selecionados 11 artigos. Os preditores da SB pode ser atribuído a múltiplos fatores, porém associados com maior prevalência ao estresse nos setores de trabalho, assim como a ações estruturais que correspondem a maior parte do relato dos profissionais e a sintomatologia dos sinais e sintomas sofrem variação de cada profissional, podendo estar relacionada a situações estressantes vivenciadas durante o trabalho. Diante das informações expostas, realizar acompanhamento psicológico com esses profissionais da saúde é oportuno, valorizando assim a saúde dos mesmo e prevenido futuras complicações.
\end{abstract}

Palavras-chave: Esgotamento psicológico; Enfermagem; Trabalho.

\begin{abstract}
This study aimed to conduct an active search in the literature on SB in nursing professionals working around urgency and emergency, addressing together the main predictors for the development of the syndrome. This is a descriptive study, with a qualitative methodology approach, using the Integrative Literature Review (ILR) as a technique, together with a spiking strategy to formulate the guiding question. The research was carried out between the months of April and June 2021 in the indexed bases within the Virtual Health Library (VHL), being: (MEDLINE), (LILACS) and (BDENF), through the junction of three descriptors in health (DeCS) crossed with the Boolean operator "AND": Psychological exhaustion AND Nursing AND Work, being found 1,007 articles in total. After applying the criteria of publications within the Portuguese and English languages, within the established timeline of the last five years (2016 to 2021), that had a correlation with the problem question, 11 articles were selected. The predictors of BS can be attributed to multiple factors, but they are associated with higher prevalence of stress in the work sectors, as well as structural actions that correspond to most of the professionals' reports and the symptoms of signs and symptoms vary from professional to professional and may be related to stressful situations experienced during work. In view of the information presented, performing psychological follow-up with these health professionals is opportune, thus valuing their health and preventing future complications.
\end{abstract}

Keywords: Psychological burnout; Nursing; Work.

\title{
Resumen
}

Este estudio tuvo como objetivo realizar una búsqueda bibliográfica activa sobre el SB en los profesionales de enfermería que trabajan en el área de urgencias y emergencias, abordando conjuntamente los principales predictores para el desarrollo del síndrome. Se trata de un estudio descriptivo, con un enfoque metodológico cualitativo, que utiliza como técnica la Revisión Integradora de la Literatura (RIL), junto con una estrategia de pico para formular la pregunta guía. La investigación se realizó entre los meses de abril y junio de 2021 en las bases indexadas dentro de la Biblioteca Virtual de Salud (BVS), siendo: (MEDLINE), (LILACS) y (BDENF), a través de la unión de tres descriptores en salud (DeCS) cruzados con el operador booleano "AND": Agotamiento Psicológico AND Enfermería AND Trabajo, encontrándose 1.007 artículos en total. Después de aplicar el criterio de las publicaciones dentro de los idiomas portugués e inglés, dentro de la línea de tiempo establecida en los últimos cinco años (2016 a 2021), que tenían una correlación con la pregunta del problema, se seleccionaron 11 artículos. Los predictores del SB pueden atribuirse a múltiples factores, pero se asocian a una mayor prevalencia del estrés en los sectores de trabajo, así como a acciones estructurales que corresponden a la mayoría de los informes de los profesionales, y la sintomatología de los signos y síntomas varía de un profesional a otro, y puede estar relacionada con las situaciones estresantes vividas durante el trabajo. Dada la información presentada anteriormente, es conveniente realizar un seguimiento psicológico con estos profesionales de la salud, valorando así su salud y previniendo futuras complicaciones.

Palabras clave: Agotamiento psicológico; Enfermería; Trabajo. 


\section{Introdução}

A experiência dicotômica das emoções em conjunto com as atividades de trabalho realizadas dentro do ambiente hospitalar contribui tanto para fatores relaxantes como estressantes. Grande parte de operações estressantes estão relacionadas principalmente aos profissionais da saúde, com maior prevalência os profissionais de enfermagem, que são responsáveis pelo contato (direto/indireto) com pacientes doentes ou que apresentam alguma complicação (acidentes, traumas e lesões), sustentando assim a proximidade com o sofrimento e desconforto desses pacientes (Barbosa et al., 2021).

Outros fatores também corroboram para sofrimento mental desses profissionais da saúde, destacando-se à baixa remuneração, onde muitos profissionais de enfermagem se sucumbem a dobrar suas horas dentro do ambiente hospitalar, cedendo assim a vários fatores particulares da vida pessoal em prol do trabalho, aumentando as chances de desenvolvimento de fatores que possam contribuir para o aparecimento de complicações envolvendo a saúde mental (Mota et al., 2020).

Mediante a estas exposições, pode ocorrer o surgimento de diversas síndromes, incluído a Síndrome de Burnout (SB), sendo ela caracterizada principalmente pelo cansaço físico e emocional atribuídos às ações desenvolvidas durante o ambiente de trabalho, gerando complicações que podem influenciar na qualidade de vida dos acometidos. A síndrome é dividida em três grandes dimensões: fadiga mental, despersonalização e frustração ocupacional (Silva et al., 2015).

A fadiga mental é caracterizada por fatores que envolvem a perda de energia, incluindo sentimentos de tristeza, frustação, impaciência, desesperança e estresse. Tais complicações auxiliam no aparecimento de sintomas de vulnerabilidade, como: náuseas, desconforto cervical e lombar, cefaleia, tensão muscular e principalmente ações que irão prejudicar o padrão do sono (Ramos et al., 2019).

A despersonalização é vista como um artefato que envolve o cuidado prestado ao paciente, correspondendo as atividades e pensamentos negativos no setor de trabalho, tratando as pessoas como objeto, interferindo assim em uma assistência imprecisa, desencadeando pares de organização que estão envolvidos com o isolamento social desse profissional no setor de trabalho (Duhtra et al., 2019).

Por último, a decepção ou esgotamento profissional, está relacionada ao sentimento de incompetência envolvendo um padrão comportamental voltado para o autojulgamento, no qual ele se sente insatisfeito com o seu sucesso dentro da sua área de atuação, interferindo assim nas ações assistenciais prestadas aos pacientes, afetando em queda da sua produtividade e a sua realização no trabalho (Russo et al., 2019).

Herbert Freudenberger (1974), definiu o termo Burnout como a síndrome da fadiga profissional que era sintomatológica, essa palavra é derivada do latim onde o "Burnout" significa consumir ou queimar inteiramente até a exaustão final. Assim, Freudenberger definiu a SB como uma correlação da sensação de perda em conjunto com a fadiga e o desgaste emocional, onde tais fatores (irritabilidade, depressão, inflexibilidade e rigidez) foram descobertos mais tarde, porém possui grande contribuição para o desenvolvimento da síndrome (Vieira et al., 2019).

Correlacionando a SB com os profissionais de enfermagem que atuam dentro da área de urgência e emergência, ela pode ocorrer com maior frequência, uma vez que determinadas situações podem ocorrer em caráter imediato ou mediato, colocando a vida das pessoas nas suas escolhas, sendo obrigados a tomarem decisões que podem salvar a vida desses pacientes em um menor tempo possível. Outros fatores também corroboram para o desenvolvimento da SB nesses profissionais, como a sobrecarga de trabalho e a rotina estressante, assim como o sofrimento dos familiares, baixo número de profissionais e falta de insumos e materiais nas unidades de saúde (Fernandes, 2015).

Diante das informações expostas na presente pesquisa, abordar sobre fatores que possam contribuir para o melhor entendimento da SB nesses profissionais é algo válido, uma vez que a síndrome é caracterizada com um grande problema de saúde que insere fatores que ocorrem em decorrência do trabalho, sendo classificada como a síndrome do esgotamento profissional, segundo a Lei $\mathrm{n}^{\mathrm{o}}$ 3.048/99, fazendo parte do grupo V, da CID (Z73.0) da previdência social no anexo II (Brasil, 
2007).

Frente a isso, a seguinte pesquisa teve por objetivo realizar uma busca ativa na literatura sobre a SB nos profissionais de enfermagem que atuam na área de urgência e emergência, abordando em conjunto os principais preditores para o seu desenvolvimento.

\section{Metodologia}

A seguinte pesquisa trata-se de um estudo de origem descritiva, com abordagem qualitativa, utilizando a Revisão Integrativa da Literatura (RIL). A RIL pode resumir o passado, seja ele teórico ou empírico, através da coleta de dados e informações de artigos publicados anteriormente em periódicos distintos com diferentes abordagens metodológicas, contribuindo assim para área pesquisada e a melhora da escrita científica (Soares et al., 2014).

Para elaboração de uma pergunta problema (norteadora) utilizou-se a estratégia PICO: onde P - população (profissionais de enfermagem que atuam na área de urgência e emergência); I - intervenção (Síndrome de Burnout); C comparação (não se aplica a este estudo, uma vez que ela não se trata de uma pesquisa comparativa); $\mathrm{O}$ - desfecho (principais preditores para o desenvolvimento da SB nesses profissionais) (Santos et al., 2007). Após isso, foi formulada a seguinte pergunta norteadora: Quais os principais preditores que contribuem para o desenvolvimento da Síndrome de Burnout nos profissionais de enfermagem que atuam na área de urgência e emergência?

A pesquisa foi desenvolvida em cinco momentos subsequentes, 1 - identificação da problemática em questão; 2 elaboração dos critérios de elegibilidade e inelegibilidade; 3 - avaliação completa das publicações selecionadas para revisão; 4 - interpretação dos artigos; 5 - síntese completa dos estudos. A metodologia aplicada para seleção dos artigos foi a leitura do título e do resumo, quando necessário a leitura completa da publicação em seleção.

A pesquisa foi realizada entre os meses de abril e junho de 2021 nas bases indexadas dentro da Biblioteca Virtual de Saúde (BVS), sendo: Sistema Online de Busca e Análise de Literatura Médica (MEDLINE), Literatura Latino-Americana e do Caribe em Ciências da Saúde (LILACS) e Base de Dados Bibliográficas Especializada na área de Enfermagem (BDENF), através da junção de três descritores em saúde (DeCS) cruzados com o operador booleano "AND": Esgotamento psicológico AND Enfermagem AND Trabalho.

Os critérios de elegibilidade foram: publicações dentro dos idiomas português e inglês, dentro da linha temporal estabelecida nos últimos cinco anos (2016 a 2021) e que apresentaram semelhança com o objetivo central da questão pesquisada. Os de exclusão foram: publicações fora dos idiomas pré-estabelecidos (português e inglês), anteriores ao ano de 2016 e que não apresentaram semelhança com a questão problema.

As bases de dados foram selecionadas por possuírem maior acessibilidade de artigos publicados em diferentes revistas indexadas, proporcionado assim uma melhor abordagem metodológica para a presente pesquisa. Artigos que necessitavam e não possuíam aprovação do comitê de ética foram descartados para evitar incongruências ou informações errôneas neste estudo.

\section{Resultados}

Utilizando os descritores "Esgotamento psicológico" AND "Enfermagem" AND "Trabalho", foram encontradas 1.007 publicações, sendo 818 no MEDLINE, 121 no LILACS e 107 na BDENF. Ao adicionar os critérios de artigos publicados dentro do corte temporal de cinco anos (2016 a 2021) nos idiomas português e inglês, este número reduziu para 172. Após a leitura, interpretação e análise das pesquisas, 11 artigos relacionados ao objetivo central, com foco na pergunta problema, foram selecionados para compor esta pesquisa (Figura 1). 
Figura 1: Fluxograma das etapas de seleção dos artigos para revisão.

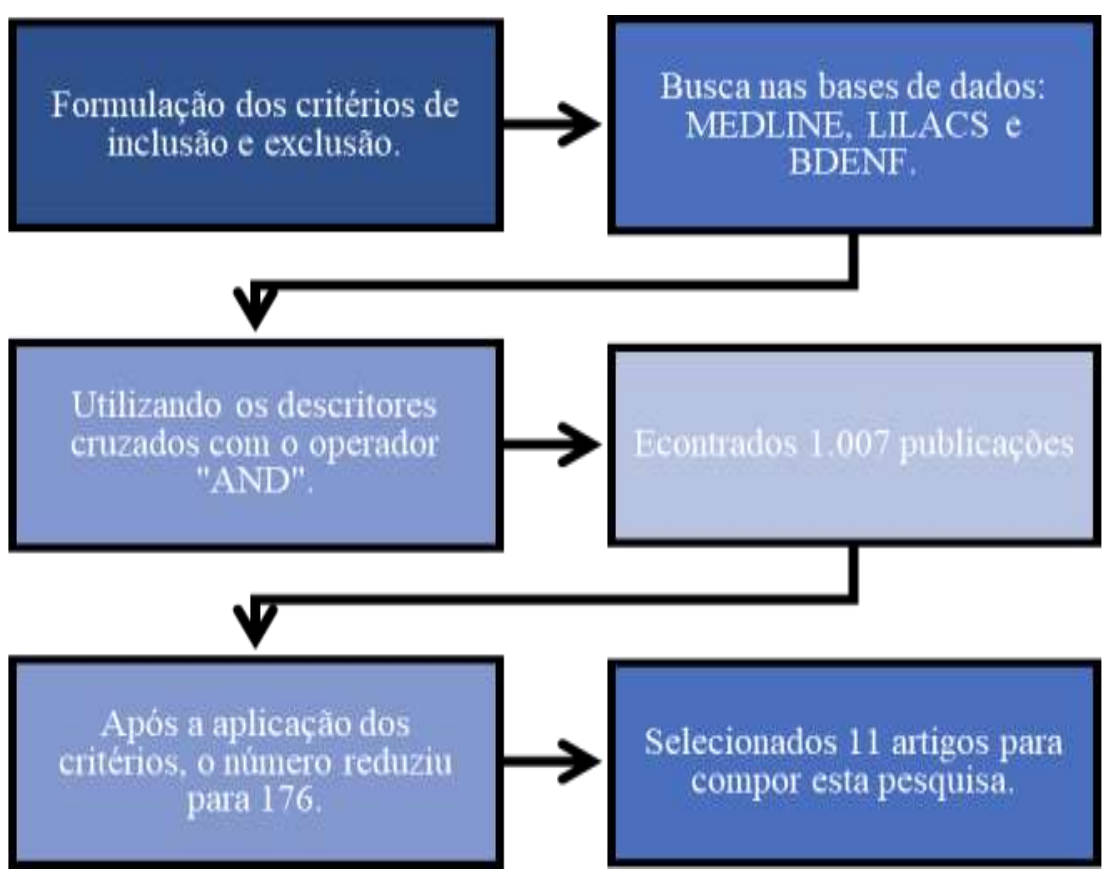

Fonte: Fabricia et al. (2021).

Para melhor organização das publicações selecionadas para compor esta revisão, será apresentada uma síntese completa das pesquisas conforme autor, ano, título, objetivo estudo e publicação, facilitando assim no processo de interpretação e análise dos estudos presentes nesta revisão integrativa (Tabela 1).

Tabela 1: Síntese completa dos estudos selecionados.

\begin{tabular}{|c|c|c|c|c|c|}
\hline $\mathbf{N}^{\circ}$ & Autor/Ano & Título & Objetivo & Estudo & Publicação \\
\hline 1 & $\begin{array}{c}\text { Gusmão et al., } \\
2019 .\end{array}$ & $\begin{array}{l}\text { Síndrome de Burnout em } \\
\text { enfermeiros que atuam no } \\
\text { serviço de urgência e } \\
\text { emergência: } \\
\text { revisão integrativa. }\end{array}$ & $\begin{array}{l}\text { Identificar, por meio de uma revisão } \\
\text { integrativa, a síndrome de Burnout em } \\
\text { enfermeiros que atuam no serviço de } \\
\text { urgência e emergência. }\end{array}$ & Descritivo & $\begin{array}{c}\text { Revista } \\
\text { Intercâmbio }\end{array}$ \\
\hline 2 & Souza, 2018. & $\begin{array}{l}\text { Síndrome de Burnout em } \\
\text { equipe de enfermagem que } \\
\text { atua na urgência e } \\
\text { emergência. }\end{array}$ & $\begin{array}{l}\text { Investigar a presença de sinais e } \\
\text { sintomas } \\
\text { de exaustão física e psicológica que } \\
\text { possam indicar a ocorrência de } \\
\text { síndrome de Burnout em profissionais } \\
\text { da equipe de enfermagem. }\end{array}$ & Exploratório & $\begin{array}{l}\text { Revista } \\
\text { Saúde } \\
\text { Coletiva }\end{array}$ \\
\hline 3 & $\begin{array}{c}\text { Coelho et al., } \\
2017 .\end{array}$ & $\begin{array}{c}\text { Síndrome de Burnout nos } \\
\text { profissionais de enfermagem } \\
\text { de emergência. }\end{array}$ & $\begin{array}{l}\text { Analisar a produção científica } \\
\text { relacionada à síndrome de Burnout nos } \\
\text { profissionais de enfermagem dos } \\
\text { serviços de emergência. }\end{array}$ & Descritivo & $\begin{array}{l}\text { International } \\
\text { Nursing } \\
\text { Congress }\end{array}$ \\
\hline 4 & $\begin{array}{l}\text { Paula et al., } \\
2020 .\end{array}$ & $\begin{array}{l}\text { Fatores que desencadeiam a } \\
\text { síndrome de Burnout em } \\
\text { Enfermeiros da emergência. }\end{array}$ & $\begin{array}{l}\text { Identificar, por meio da literatura } \\
\text { brasileira, fatores relacionados à } \\
\text { síndrome de Burnout em enfermeiros } \\
\text { que trabalham na emergência }\end{array}$ & Descritivo & $\begin{array}{l}\text { Revista de } \\
\text { iniciação } \\
\text { científica e } \\
\text { extensão }\end{array}$ \\
\hline 5 & Silva, 2017. & $\begin{array}{l}\text { O estresse ocupacional e a } \\
\text { equipe de enfermagem de } \\
\text { urgência / emergência: um } \\
\text { olhar para estudos brasileiros }\end{array}$ & $\begin{array}{l}\text { Analisar a produção científica } \\
\text { relacionada ao estresse ocupacional que } \\
\text { a equipe de enfermagem de urgência } \\
\text { e/ou emergência está exposta }\end{array}$ & Descritivo & $\begin{array}{l}\text { Instituto de } \\
\text { Ciências } \\
\text { Básicas da } \\
\text { Saúde }\end{array}$ \\
\hline 6 & $\begin{array}{c}\text { Oliveira, } \\
2019 .\end{array}$ & $\begin{array}{l}\text { A síndrome de Burnout entre } \\
\text { enfermeiros do setor de } \\
\text { urgência e emergência: uma } \\
\text { revisão narrativa }\end{array}$ & $\begin{array}{l}\text { Identificar os fatores que desencadeiam } \\
\text { a Síndrome de Burnout ente } \\
\text { enfermeiros no âmbito da urgência e } \\
\text { emergência }\end{array}$ & Descritivo & $\begin{array}{l}\text { Centro } \\
\text { Universitário } \\
\text { de Brasília }\end{array}$ \\
\hline
\end{tabular}


Research, Society and Development, v. 10, n. 7, e20910716372, 2021

(CC BY 4.0) | ISSN 2525-3409 | DOI: http://dx.doi.org/10.33448/rsd-v10i7.16372

\begin{tabular}{|c|c|c|c|c|c|}
\hline 7 & $\begin{array}{l}\text { Portela et al., } \\
2015 .\end{array}$ & $\begin{array}{c}\text { Síndrome de Burnout em } \\
\text { profissionais de enfermagem } \\
\text { de serviços de urgência e } \\
\text { emergência }\end{array}$ & $\begin{array}{l}\text { Analisar como os estudos científicos } \\
\text { descrevem a síndrome de Burnout em } \\
\text { profissionais de } \\
\text { enfermagem de serviços de urgência e } \\
\text { emergência }\end{array}$ & Descritivo & $\begin{array}{l}\text { Revista de } \\
\text { Pesquisa } \\
\text { Cuidado é } \\
\text { Fundamental } \\
\text { Online }\end{array}$ \\
\hline 8 & Lordão, 2019. & $\begin{array}{l}\text { Síndrome de Burnout na } \\
\text { equipe de enfermagem em } \\
\text { unidades de pronto } \\
\text { atendimento }\end{array}$ & $\begin{array}{c}\text { Verificar a produção científica em } \\
\text { Síndrome de Burnout na Enfermagem } \\
\text { no período de janeiro de } 2000 \text { a } \\
\text { dezembro de } 2018\end{array}$ & Bibliométrico & $\begin{array}{l}\text { Universidade } \\
\text { Federal da } \\
\text { Paraíba }\end{array}$ \\
\hline 9 & $\begin{array}{l}\text { Pinhal et al., } \\
2018 .\end{array}$ & $\begin{array}{l}\text { Fatores de risco da síndrome } \\
\text { de Burnout entre enfermeiros } \\
\text { da } \\
\text { Urgência e emergência }\end{array}$ & $\begin{array}{c}\text { descrever sobre os fatores de risco da } \\
\text { síndrome de Burnout entre enfermeiros } \\
\text { da } \\
\text { urgência e emergência no âmbito } \\
\text { hospitalar. }\end{array}$ & Descritivo & $\begin{array}{l}16^{\circ} \\
\text { Seminário de } \\
\text { Pesquisa }\end{array}$ \\
\hline 10 & $\begin{array}{l}\text { Zafra et al., } \\
2016\end{array}$ & $\begin{array}{c}\text { Síndrome de Burnout } \\
\text { relacionada aos } \\
\text { Funcionários das unidades de } \\
\text { pronto } \\
\text { Atendimento em maringá, } \\
\text { paraná, brasil }\end{array}$ & $\begin{array}{l}\text { Identificar a existência da Síndrome de } \\
\text { Burnout entre os profissionais de } \\
\text { enfermagem nas Unidades de Pronto } \\
\text { Atendimento do município de Maringá. }\end{array}$ & Exploratório & $\begin{array}{l}\text { Revista } \\
\text { UNINGÁ }\end{array}$ \\
\hline 11 & $\begin{array}{l}\text { Lopes et al., } \\
2020 .\end{array}$ & $\begin{array}{l}\text { Síndrome de Burnout e os } \\
\text { seus Efeitos sobre a Vida dos } \\
\text { Profissionais de Enfermagem } \\
\text { da Urgência e Emergência }\end{array}$ & $\begin{array}{l}\text { analisar a Síndrome de Burnout e seus } \\
\text { efeitos apresentados na vida dos } \\
\text { profissionais de enfermagem de } \\
\text { serviços de urgência e emergência }\end{array}$ & Descritivo & $\begin{array}{l}\text { Revista de } \\
\text { iniciação } \\
\text { científica e } \\
\text { extensão. }\end{array}$ \\
\hline
\end{tabular}

Fonte: Fabricia et al. (2021).

Em relação aos tipos de estudos selecionados, oito $(72,8 \%)$ são do tipo descritivo, dois $(18,1 \%)$ exploratório e um bibliométrico $(9,1 \%)$, sendo a abordagem qualitativa presente em todas as pesquisas. No presente artigo não houve revista ou periódico dominante, porém com maiores contribuições tivemos a Revista de Iniciação Científica e extensão com duas publicações no ano de 2020 (Gráfico 1).

Gráfico 1: Tipos de estudos encontrados.

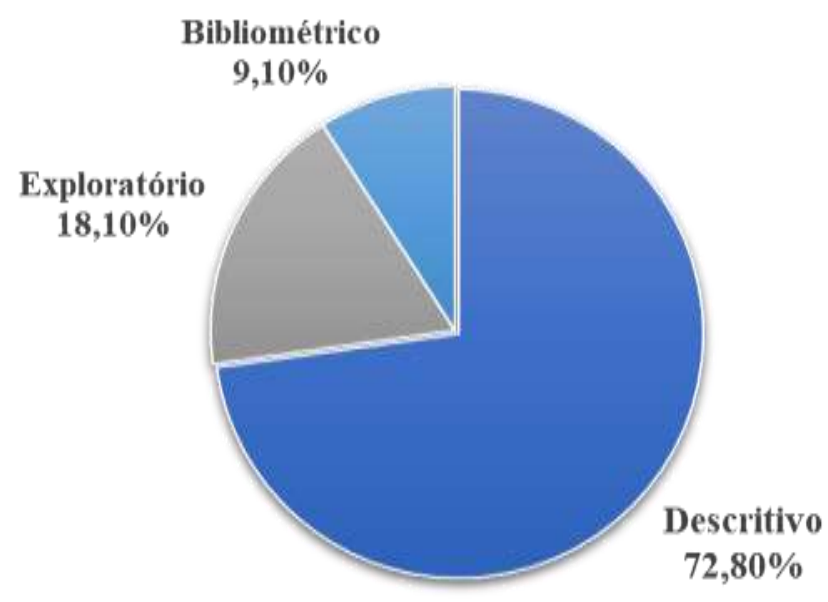

- Descritivo Exploratório 回Bibliométrico

Fonte: Fabricia et al. (2021). 


\section{Discussão}

\subsection{Principais preditores para SB em enfermeiros que atuam na urgência e emergência}

Os preditores da SB podem ser atribuídos a múltiplos fatores, porém associados com maior prevalência ao estresse nos setores de trabalho, assim como às ações estruturais que correspondem a maior parte do relato dos profissionais. Na pesquisa de Coelho et al. (2017), o barulho, falta de materiais, sobrecarga de serviço, diminuição dos números de profissionais, dupla jornada de trabalho e a baixa remuneração são os principais fatores que favorecem para o desenvolvimento da SB nos profissionais de enfermagem que atuam na área de urgência e emergência.

Segundo Gusmão et al. (2019), a SB acontece principalmente nos profissionais de enfermagem que atuam na urgência e emergência pelos fatores estressantes (intrínsecos e extrínsecos) ocasionados no setor de trabalho, sendo observado em um estudo realizado no hospital de Emergência em Campina Grande com 40 profissionais de enfermagem que a baixa remuneração é o principal fator responsável por desencadear a síndrome.

Lopes et al. (2020) abordam em sua pesquisa um estudo realizado com 32 profissionais atuantes no Serviço de Atendimento Móvel de Urgência (SAMU) no município de Picos - Piauí, onde destacou que o principal fator que contribui para o desenvolvimento da SB nesses profissionais é a jornada de trabalho de até 40 horas semanais, sendo verificado que metade $(41,7 \%)$ dos enfermeiros trabalhavam mais de 60 horas por semana, estando assim sujeitos a alto nível de desgaste emocional e físico.

Diferente dos principais preditores já citados acima, de acordo com a pesquisa de Portela et al. (2015) o principal fator de risco para o desenvolvimento da SB nesses profissionais é a dupla ou maior jornada de trabalho, onde os mesmos ao assumir vários turnos renunciam a suas atribuições e prazeres pessoais em prol do trabalho, ficando assim mais susceptível ao desencadeamento de múltiplos fatores estressantes.

No estudo de Zafra et al. (2016) onde foi realizado uma pesquisa no hospital de Emergência em Campina Grande com 40 profissionais de enfermagem, foi observado que a baixa remuneração é o principal fator para o desenvolvimento da SB, apontando assim pontos de desequilíbrio entre a companhia e trabalhador. Entretanto, no estudo de Souza (2018) destacou-se ações voltadas para possuir funções que exigem mais tempo do que o trabalhador se julga capaz de conseguir executar, levando o mesmo a torna-se irritável e deprimido.

\subsection{Sinais aparentes da SB em enfermeiros que atuam na área de urgência e emergência}

A sintomatologia sofre variação de cada profissional, podendo estar relacionada principalmente a situações estressantes vivenciadas durante o trabalho. Entretanto, os principais sinais identificados nesses profissionais são: cefaleia, tensão muscular, sensação de fadiga, problemas de memória e insônia. Relacionado aos problemas psicológicos, foram observados com maior destaque: diminuição da libido, alterações de humor e sensibilidade emocional excessiva (Coelho et al., 2017).

Pinhal et al. (2018) em sua pesquisa realizada com 30 profissionais de atuam no SAMU no estado de Fortaleza (CE) apenas 9 apresentavam sinais e sintomas de estresse, com maior prevalência de manifestações psicológicas. Entretanto no estudo de Lordão et al. (2019) realizado no pronto-socorro de um hospital no Paraná, observaram que 61 dos profissionais pesquisados, somente $8,2 \%$ apresentaram sinais de estresse (avançado), e 54,41\% possuíam uma maior chance de desenvolver a SB nos próximos anos.

No estudo de Souza (2018), os primeiros sinais e sintomas aparentes da síndrome nos profissionais de enfermagem são: alterações gastrointestinais, absenteísmo, cefaleia, intenção de sair do emprego, aumento da rotatividade, baixa produtividade e diminuição da satisfação com o trabalho.

Na pesquisa de Lopes et al. (2020) sinais como: atitudes frias, negativas e irônicas são ações que possuem grande 
relação com a SB nesses profissionais. Os sinais aparentes são desenvolvidos em decorrência dos fatores estressantes (extrínsecos e intrínsecos) no setor de trabalho.

Segundo Gusmão et al. (2019), esses profissionais incialmente apresentam os seguintes sinais (constante e progressivo): fadiga, dores musculares e ósseas, inquietações gastrointestinais, insônia, cefaleia, imunodeficiência, distúrbios respiratórios, disfunções sexuais e alterações menstruais nas mulheres. Por outro lado, em relação aos psicológico, ocorre a perda da memória, solidão, irritação, baixa autoestima e desânimo, podendo levar a dependência de medicamentos.

\subsection{Fatores sociodemográficos e ocupacionais relacionados a SB em enfermeiros}

A SB pode se apresentar em qualquer profissional, porém os profissionais que atuam na área de enfermagem, com foco no serviço de urgência e emergência possuem uma maior predisposição de adquirir a doença. Em relação aos fatores socioambientais da SB, foi observado que esses profissionais se tornam mais expostos aos riscos envolvendo o estresse ocupacional, estando relacionado a recursos humanos impróprios e apresentarem possíveis inseguranças na melhor conduta (Zafra et al., 2016).

Segundo Paula et al. (2020) em relação a idade, os profissionais de enfermagem que atuam na área da urgência e emergência mais acometidos são os jovens, acredita-se que isso possa ocorrer principalmente por se sentirem despreparados, quando a maioria ainda é residente, além de possuírem também uma carga de trabalho exaustiva durante plantões noturnos. Outras pesquisas abordam que a realização de atividade física contribui na melhora da condição de saúde do trabalhador, reduzindo assim às tensões e estresses adquiridos no setor de trabalho.

A superlotação dos ambientes de urgência e emergência é frequente, contribuindo para o problemas mentais nesses profissionais, não somente pela sobrecarga de trabalho, mas também pela maior demanda de atribuições, que na maioria das vezes trabalham com a equipe reduzida, isso eleva grandiosamente o estresse, alimentando o desgaste físico e emocional, favorecendo assim para o aumento de chances de possíveis erros e danos no setor de trabalho, com maior prevalência os eventos adversos, colocando em risco a vida dos pacientes (Pinhal et al., 2018).

As atividades administrativas ou relacionadas a gerência do setor também são consideradas aspectos estressantes para os enfermeiros que atuam nessa área, uma vez que além de serem responsáveis pelo cuidado desses pacientes, eles também precisam gerenciar a equipe de enfermagem, através de ações como: supervisionar as atividades da equipe durante o período de trabalho e realizar a distribuição de funcionários por meio das escalas. Dessa forma, as atividades gerenciais exercidas pelo enfermeiro seja ela administrativa ou não também tem sido um grande fator de estresse responsável pelo desenvolvimento da SB nesses profissionais (Pinhal et al., 2018).

Segundo Portela et al. (2015) a exposição a perfurocortantes e alguns materiais contaminados também são geradores de estresse, uma vez que durante a assistência os procedimentos são realizados com rapidez, tornando os profissionais mais susceptíveis a essa contaminação por material biológico. O barulho também foi abordado na pesquisa, sendo citado que a repetitividade de ruídos é algo comum na área de urgência e emergência, fazendo com que o organismo libere corticoide, noradrenalina e adrenalina, elevando assim os níveis de estresse nesses profissionais.

A falha na comunicação entre os profissionais é algo abordado com frequência nas pesquisas, sendo um dos fatores que favorecem nesse processo. Foi observado que essa falha interfere principalmente na assistência prestada ao paciente e na qualidade das ações prestadas para a saúde dos assistidos, favorecendo o surgimento da SB (Coelho et al., 2017).

Pinhal (2018) aborda em sua pesquisa que as mulheres são mais susceptíveis ao desenvolvimento da SB, estando relacionada principalmente a não conseguirem conciliar a jornada de trabalho com as tarefas domiciliares, comprometendo assim seu sono, descanso e lazer. Entretanto, outras pesquisas demostram que indivíduos solteiros que ainda moram com seus familiares possuem uma menor chance de desenvolver a síndrome. 
Na pesquisa de Lopes et al. (2020) o predomínio relacionado as dimensões da síndrome abordaram diferentes percentuais nos profissionais de enfermagem que atuam na urgência e emergência em ambos os sexos, sendo o desgaste emocional com maior prevalência no sexo masculino $(70,8 \%)$, a despersonalização $(87,5 \%)$ com maior índice em mulheres e, o esgotamento profissional $(87,5 \%)$ também nas mulheres. A pesquisa não apresentou similaridade de percentual em ambos os sexos.

Segundo Portela et al. (2015) a síndrome se apresenta de forma disfarçada nas mulheres, sendo correlacionada com as situações vivenciadas no setor de trabalho e em casa, verificou-se que $(46,9 \%)$ são casadas e $(56,3 \%)$ possuem um ou mais filhos, atribuindo assim o gênero feminino com maior aptidão para o desenvolvimento da SB. Em relação a escolaridade, a pesquisa mostrou que quanto maior for a sua titulação, maior é o seu nível de estresse, corroborando ainda mais para a SB.

\subsection{Intervenção e prevenção da síndrome de Burnout}

A SB é considerada uma doença ocupacional que afeta principalmente os profissionais em seu setor de trabalho, levando os mesmo a se afastarem, gerando grandes prejuízo para o empregador. Torna-se urgente a implantação de pesquisas, estudos e ações que valorizem a saúde mental desses profissionais de enfermagem que atuam na área de urgência e emergência, evidenciando medidas preventivas e públicas para que essas entrem em vigor (Mourão, 2017).

Estudos que envolvam ações de prevenção e intervenção a SB nesses profissionais continuam obsoletos, propostas vêm sendo inseridas no sistema de saúde, como: estruturação do tempo livre com atividades prazerosas e atrativas, busca de convivência menos conflituosa com pares e grupos, organizações de trabalho, avaliação periódica da qualidade de vida individual, avaliação do limite individual de tolerância e exigência, aprimoramento do conhecimento de seus problemas de saúde, sociais e econômicos (Silveira, 2016).

\section{Considerações Finais}

Diante das informações apresentadas, a dupla jornada de trabalho se mostrou um dos principais preditores para o desenvolvimento da síndrome de Burnout nesses profissionais de enfermagem, apresentando sinais como: insônia, desânimo e apatia com maior incidência. Logo, é clara a necessidade de prestar atenção no bem-estar físico, mental e emocional desses profissionais, assim como seus fatores sociodemográficos e ocupacionais que podem favorecer para o desenvolvimento da síndrome. Diante do exposto, realizar acompanhamento psicológico com esses profissionais da saúde é oportuno, valorizando assim a saúde e prevenindo futuras complicações.

\section{Referências}

Barbosa, I. E. B., Fonseca, A. R., de Andrade, E. N. M., Maklouf, D. C., Ribeiro, M. C. S., da Silva Rodrigues, A. J. P., \& de Figueiredo Lira, F. C. (2021). Síndrome de burnout: fatores sociodemográficos e ocupacionais em profissionais da enfermagem. Revista Eletrônica Acervo Saúde, 13 (3), e6618-e6618.

Bardaquim, V. A., Dias, E. G., de Marchi Barcellos, R. D. C., \& Robazzi, M. L. D. C. C. (2019). Reflexão sobre as condições de trabalho da enfermagem: subsídio às 30 horas de trabalho. Revista Enfermagem Contemporânea, 8(2), 172-181.

Coelho, L. M., \& Silva, E. F. L. (2017, December). Síndrome de Burnout nos profissionais de enfermagem de emergência. In Congresso Internacional de Enfermagem (Vol. 1, No. 1).

da Cruz Soares, S. G., Gomes, M. R. S., \& de Oliveira Araujo, M. (2020). Relação entre condições de trabalho e saúde do enfermeiro emergencista. Revista de Enfermagem e Atenção à Saúde, 9(2).

das Merces, M. C., Lopes, R. A., de Souza, D., Oliveira, D. S., Lua, I., Mattos, A. I. S., \& Júnior, A. D. O. (2017). Prevalência da Síndrome de Burnout em profissionais de enfermagem da atenção básica à saúde Prevalence of Burnout Syndrome in nursing professionals of basic health care. Revista de Pesquisa: Cuidado é Fundamental Online, 9(1), 208-214. 
de Oliveira, L. P. S., \& Araújo, G. F. (2016). Características da síndrome de burnout em enfermeiros da emergência de um hospital público. Revista Enfermagem Contemporânea, 5(1).

de Sousa, H. R. O. (2018). Síndrome de Burnout em equipe de enfermagem que atua na urgência e emergência. Tempus Actas de Saúde Coletiva, 11(4), ág185 .

de Souza Mota, B., Figueiredo, S. N., Siqueira, D. S. G., Queiroz, N. D. A., \& dos Santos, T. M. D. P. (2020). As contribuições da síndrome de burnout para o déficit do trabalho da enfermagem: revisão integrativa da literatura. Revista Eletrônica Acervo Saúde, 12(10), e4383-e4383.

de Souza Pereira, S., de Castro Silva, P. M., de Azevedo, E. B., Faustino, E. B., Nicolau, Z. M., \& Filha, M. D. O. F. (2014). Síndrome de burnout em profissionais de enfermagem de um hospital de urgência/emergência. Revista da Universidade Vale do Rio Verde, 12(1), 636-647.

Fernandes, J. (2017). Estresse ocupacional, vulnerabilidade e estratégias de enfrentamento: intervenção em serviço de atendimento móvel de urgência. [dissertation]. Bauru: Universidade Estadual Paulista.

Ferreira, C. A. A., Neto, M. T. R., Kilimnik, Z. M., \& dos Santos, A. S. (2016). O Contexto do Estresse Ocupacional dos Trabalhadores da Saúde: estudo bibliométrico. Revista de Gestão em Sistemas de Saúde, 5(2), 84-99.

Gusmão, A. O. S., Teixeira, T. F. S., \& Barbosa, J. M. G. (2019). Síndrome de Burnout em enfermeiros que atuam no serviço de urgência e emergência: revisão integrativa. Revista Intercâmbio, 16, 3-14.

Lordão, A. V. (2019). Síndrome de Burnout na equipe de enfermagem em unidades de pronto atendimento. [dissertation]. João Pessoa: Universidade Federal da Paraíba.

Oliveira, L. E. L. D. (2019). A Síndrome de Burnout entre enfermeiros do setor de urgência e emergência: uma revisão narrativa. [dissertation]. Brasília: Faculdade de Ciências da Educação e Saúde.

Oliveira, P. R. C., Melo, T. G., Lopes, A., Lima, M. N., Zorzetto Filho, D., \& Carvalho, V. O. (2019). Frequência da Síndrome de Burnout em médicos residentes. Revista Residência Pediátrica, 9(2), 91-96.

Paiva, J. D. M., Cordeiro, J. J., Silva, K. K. M. D., Azevedo, G. S. D., Bastos, R. A. A., Bezerra, C. M. B., \& Martino, M. M. F. D. (2019). Fatores desencadeantes da síndrome de burnout em enfermeiros. Rev. Enferm. UFPE online, 483-490.

Paula, E. P. C. D., Valviesse, H. C., Azevedo, T. F., \& Silva, T. S. D. (2021). Fatores que desencadeiam a síndrome de burnout em enfermeiros da emergência. Pesquisa \& educação a distância, (19).

Pêgo, F. P. L., \& Pêgo, D. R. (2016). Síndrome de burnout. Rev. bras. med. trab., 171-176.

Pinhal, A. P., Borba, E. L. L., Andrei, J., \& Luvizotto, J. Fatores de risco da síndrome de burnout entre enfermeiros da urgência e emergência. Pró-Reitora de Pós-Graduação, Pesquisa e Extensão, 35.

Portela, N. L. C., de Oliveira Pedrosa, A., Cunha, J. D. S., Monte, L. R. S., Gomes, R. N. S., \& Lago, E. C. (2015). Síndrome de Burnout em profissionais de enfermagem de serviços de urgência e emergência. Revista de Pesquisa Cuidado é Fundamental Online, 7(3), 2749-2760.

Silva, C. S. D. (2017). O estresse ocupacional e a equipe de enfermagem de urgência/emergência: um olhar para estudos brasileiros. [dissertation]. Porto Alegre: Instituto de Ciências Básicas da Saúde da Universidade Federal do Rio Grande do Sul.

Tavares, K. F. A., Souza, N. V. D. D. O., Silva, L. D. D., \& Kestenberg, C. C. F. (2014). Ocorrência da síndrome de Burnout em enfermeiros residentes. Acta Paulista de Enfermagem, 27(3), 260-265.

Zafra, M. C., \& pendloski, J. (2016). Síndrome de burnout relacionada aos funcionários das unidades de pronto atendimento em maringá, paraná, brasil. Revista Uningá, 47(2).

Zomer, F. B., \& Gomes, K. M. Síndrome de burnout e estratégias de enfrentamento em profissionais de saúde: uma revisão não sistemática. Revista de Iniciação Científica, 15(1). 